\title{
Thioredoxin and aging: What have we learned from the survival studies?
}

\author{
Madeline G. Roman ${ }^{\mathrm{a}}$, Lisa C. Flores ${ }^{\mathrm{a}}$, Geneva M. Cunningham ${ }^{\mathrm{a}}$, Christie Cheng ${ }^{\mathrm{a}}$, Colton Allen ${ }^{\mathrm{a}}$, \\ Gene B. Hubbard ${ }^{\mathrm{a}, \mathrm{b}}$, Yidong Bai ${ }^{\mathrm{c}}$, Thomas L. Saunders ${ }^{\mathrm{e}}$, Yuji Ikeno ${ }^{\mathrm{a}, \mathrm{b}, \mathrm{d}^{*}}$
}

\author{
${ }^{a}$ Barshop Institute for Longevity and Aging Studies, The University of Texas Health Science Center at San Antonio, San Anto- \\ nio, TX, USA \\ ${ }^{b}$ Department of Pathology, The University of Texas Health Science Center at San Antonio, San Antonio, TX, USA \\ ${ }^{c}$ Department of Cell Systems \& Anatomy, The University of Texas Health Science Center at San Antonio, San Antonio, TX, USA \\ ${ }^{d}$ Geriatric Research and Education Clinical Center, Audie L. Murphy VA Hospital, South Texas Veterans Health Care System, \\ San Antonio, TX, USA \\ e Transgenic Animal Model Core, University of Michigan, Ann Arbor, MI, USA
}

\begin{abstract}
Our laboratory has conducted the first systematic survival studies to examine the biological effects of the antioxidant protein thioredoxin (Trx) on aging and age-related pathology. Our studies with C57BL/6 mice overexpressing $\operatorname{Trx} 1\left[\operatorname{Tg}(\text { act-TRX1 })^{+/ 0}\right.$ and $\left.\operatorname{Tg}(\mathrm{TXN})^{+/ 0}\right]$ demonstrated a slight extension in early lifespan compared to wild-type (WT) mice; however, no significant effects were observed in the later part of life. Overexpression of Trx2 in male C57BL/6 mice $\left[\mathrm{Tg}(\mathrm{TXN} 2)^{+/ 0}\right]$ demonstrated a slightly extended lifespan compared to WT mice. The pathology results from two lines of Trx1 transgenic mice showed a slightly higher incidence of age-related neoplastic diseases compared to WT mice, and a slight increase in the severity of lymphoma, a major neoplastic disease, was observed in Trx2 transgenic mice. Together these studies indicate that Trx overexpression in one compartment of the cell (cytosol or mitochondria alone) has marginal beneficial effects on lifespan. On the other hand, down-regulation of Trx in either the cytosol (Trx1KO) or mitochondria (Trx2KO) showed no significant changes in lifespan compared to WT mice, despite several changes in pathophysiology of these knockout mice. When we examined the synergetic effects of overexpressing Trx1 and Trx2, TXNTg x TXN2Tg mice showed a significantly shorter lifespan with accelerated cancer development compared to WT mice. These results suggest that synergetic effects of Trx overexpression in both the cytosol and mitochondria on aging are deleterious and the development of age-related cancer is accelerated. On the other hand, we have recently found that down-regulation of Trx in both the cytosol and mitochondria in Trx1KO x Trx2 KO mice has beneficial effects on aging. The results generated from our lab along with our ongoing study using Trx1KO $\mathrm{x}$ Trx $2 \mathrm{KO}$ mice could elucidate the key pathways (i.e., apoptosis and autophagy) that prevent accumulation of damaged cells and genomic instability leading to reduced cancer formation.
\end{abstract}

Keywords: Thioredoxin, transgenic mouse, knockout mouse, cancer, lifespan, aging

\section{Thioredoxin and aging}

Initially discovered in the early 1960 s, Trx is a small protein $(12 \mathrm{kDa})$ that acts as a reductant for a variety of

\footnotetext{
* Corresponding author: Yuji Ikeno

Mailing address: Barshop Institute for Longevity and Aging Studies, The University of Texas Health Science Center at San Antonio, 15355 Lambda Drive, San Antonio, TX 78245-3207, USA. Email: ikeno@uthscsa.edu

Received: 03 June 2020 / Accepted: 11 September 2020
}

enzymes [1-7]. It contains two redox-active cysteine residues in the active center (Cys-Gly-Pro-Cys). In humans, two forms of protein have been identified: one in cytosol (Trx1) [8] and another in mitochondria (Trx2) [9]. Trx is important in maintaining a reduced intracellular environment, which is accomplished through thiol-disulfide exchange reactions [1]. This unique feature allows Trx to efficiently control protein function by altering the redox state of structural or catalytic SH groups. Therefore, Trx may have an important function in development of agerelated pathophysiological changes through attenuation of oxidative stress/damage or alteration of redox state in various signaling pathways. 
Previous studies conducted in Caenorhabditis elegans and Drosophila melanogaster have shown that Trx plays important roles in oxidative stress/damage and longevity $[10,11]$. However, the exact roles of Trx in aging and agerelated diseases in mammals and its effect on crucial signaling pathways have not been fully explored. Therefore, our laboratory has conducted the first systematic study to examine the effects of Trx on survival using mice overexpressing or down-regulating Trx 1 (cytosol) and Trx2 (mitochondria).

Here, we review the survival studies conducted in our laboratory and discuss what we have learned about the effects of Trx on aging and age-related changes.

\section{Transgenic mice/rats overexpressing thiore- doxin 1 (cytosol)}

The first survival study to test the effects of Trx 1 overexpression was conducted using a transgenic mouse model generated with human TRX1 cDNA and the $\beta$-actin promoter $\left[\mathrm{Tg}(\text { act-TRX1) })^{+/ 0}\right]$ [12]. An extension in lifespan and increased resistance to oxidative stress were observed in $\operatorname{Tg}(\text { act-TRX } 1)^{+/ 0}$ mice compared to WT mice $[13,14]$. Although these observations were very exciting, it is worth noting that the lifespan of WT mice (C57BL/6) in this study (approximately 23 months) was considerably shorter than expected from other studies using C57BL/6 mice housed in barrier facilities, which suggests that conditions for the survival experiment were not optimal. The median lifespan of C57BL/6 mice in our aging colonies, for example, is approximately $29-30$ months. Thus, it was necessary to conduct an additional survival study using $\operatorname{Tg}(\text { act-TRX } 1)^{+/ 0}$ mice to determine the effects of overexpressing Trx 1 under defined pathogen-free housing conditions.

Our study showed that Trx 1 overexpression in $\operatorname{Tg}($ actTRX 1$)^{+/ 0}$ mice resulted in a higher resistance to oxidative stress and lower levels of oxidative damage to proteins and lipids [15]. However, our survival study examining male $\mathrm{Tg}(\text { act-TRX } 1)^{+/ 0}$ mice indicated a significant life-extension only during the first half of their lifespan compared to WT mice, and no significant changes were observed thereafter. To further support these initial observations, we conducted another survival study with both male and female mice [15].

To determine why beneficial effects of Trx 1 overexpression were observed only early in life, we examined whether: 1) the levels of Trx 1 changed during aging; and 2) Trx 1 overexpression altered age-related pathology. Our data demonstrated that $\operatorname{Tg}(\text { act-TRX1 })^{+/ 0}$ mice showed significant age-related decline of Trx 1 overexpression as well as less reduction in protein oxidation levels in the later part of life [15]. This result is likely explained by the use of the $\beta$-actin promoter to control decreased expression of the transgene with age. End-of-life pathology data for these mice showed that: 1) the incidence of lung inflammation was significantly reduced in young
$\operatorname{Tg}(\operatorname{act}-\mathrm{TRX} 1)^{+/ 0}$ mice; and 2) the incidence of total fatal tumors and lymphomas were slightly higher in old $\operatorname{Tg}(\text { act-TRX } 1)^{+/ 0}$ mice compared to WT littermates [15]. Therefore, $\operatorname{Tg}\left(\right.$ act-TRX1) ${ }^{+/ 0}$ mice significantly increased in survival only during the first half of their lifespan because of an age-related reduction of Trx1 overexpression and/or enhanced tumor formation in old mice [15].

Next, we generated a new line of Trx 1 transgenic mice $\left(\mathrm{Tg}(\mathrm{TXN})^{+/ 0}\right)$ in order to determine whether continuous overexpression of Trx1 throughout life could extend maximum lifespan. These mice were generated using a fragment of the human genome containing the TXN gene [16]. $\operatorname{Tg}(\mathrm{TXN})^{+/ 0}$ mice showed significantly higher (approximately $20 \%$ to $40 \%$ ) levels of Trx 1 in the tissues than WT mice. Trx 1 overexpression in $\operatorname{Tg}(\mathrm{TXN})^{+/ 0}$ mice was maintained up to at least 28-30 months-of-age. The study showed that the survival curve of $\operatorname{Tg}(\mathrm{TXN})^{+/ 0}$ mice was not significantly different from WT mice. Although the early part of lifespan (75\% survival) showed a $6.3 \%$ extension compared to WT mice, no significant life-extending effect was observed over the lifespan [16]. Therefore, our survival data obtained from $\operatorname{Tg}(\mathrm{TXN})^{+/ 0}$ mice indicate that continuous Trx 1 overexpression in mice had some benefits only in the early part of life. This observation is similar to the effect of Trx 1 overexpression in the $\operatorname{Tg}(\text { act-TRX } 1)^{+/ 0}$ mice.

As mentioned above, the incidence of lung inflammation in young $\operatorname{Tg}(\text { act-TRX } 1)^{+/ 0}$ mice was significantly less than WT mice, and the incidence of total fatal tumors and lymphomas in old $\operatorname{Tg}(\text { act-TRX } 1)^{+/ 0}$ mice was slightly higher than WT mice [15]. These observations are consistent with some of the biological effects of Trx1, i.e., anti-inflammatory and anti-apoptotic effects. Our previous study showed lower IL-1 $\beta$ mRNA levels in the liver of Tg(act-TRX1) ${ }^{+/ 0}$ mice than WT littermates [15], which could be a contributing factor in the reduction of lung inflammation in $\mathrm{Tg}$ (actTRX 1$)^{+/ 0}$ mice. Additionally, increased levels of the ASK $1 /$ Trx1 complex were observed in $\operatorname{Tg}\left(\right.$ act-TRX1) ${ }^{+/ 0}$ mice [15], which causes inhibition of the apoptosis signal-regulating kinase-1 (ASK1) pathway [17, 18]. Therefore, Trx1 overexpression in young mice could be beneficial because of resistance to environmental stresses, including oxidative stress, and reduced inflammatory changes in the tissues, including lung. On the other hand, Trx1 overexpression in old mice may be deleterious because of its anti-apoptotic action by ASK1 inhibition and promoting cell proliferation, both of which could promote the growth of various cancers, including lymphoma [8, 19].

In addition to the studies with mice overexpressing Trx1, we also conducted a survival study with Trx 1 transgenic rats, which were generated using a fragment of the human genome containing the TXN gene as described above. We generated transgenic rats using F344 rats because they are commonly used for aging studies and could allow us to examine the possible species differences of the role of Trx1 in aging. Our data showed that the levels of Trx 1 were significantly higher [approximately 20\% to $40 \%$ : similar to $\operatorname{Tg}(\mathrm{TXN})^{+/ 0}$ mice] in all tissues examined 
in the $\operatorname{Tg}(\mathrm{TXN})^{+/ 0}$ rats compared to their WT littermates, and overexpression of $\operatorname{Trx} 1$ was maintained up to 28-30 months of age. Although $\operatorname{Tg}(\mathrm{TXN})^{+/ 0}$ rats showed similar $\operatorname{Trx} 1$ overexpression to $\operatorname{Tg}(\mathrm{TXN})^{+/ 0}$ mice, the survival curve of $\operatorname{Tg}(\mathrm{TXN})^{+/ 0}$ rats was similar to WT rats, i.e., $\operatorname{Trx} 1$ overexpression had little beneficial effects on longevity in F344 rats (Figure 1).

Overall, the survival studies described above, which were conducted with two lines of Trx 1 transgenic mice, indi- showing no changes in lifespan [20]. To further support the importance of maintaining mitochondrial functions on pathophysiology, studies have shown that mitochondrial Trx overexpression: 1) attenuated vascular dysfunction and hypertension development [21], and 2) maintained endothelial function and protected against atherosclerosis development [22]. Thus, we conducted a survival study to test whether mitochondrial Trx overexpression could show beneficial effects on aging and/or age-related diseases us-

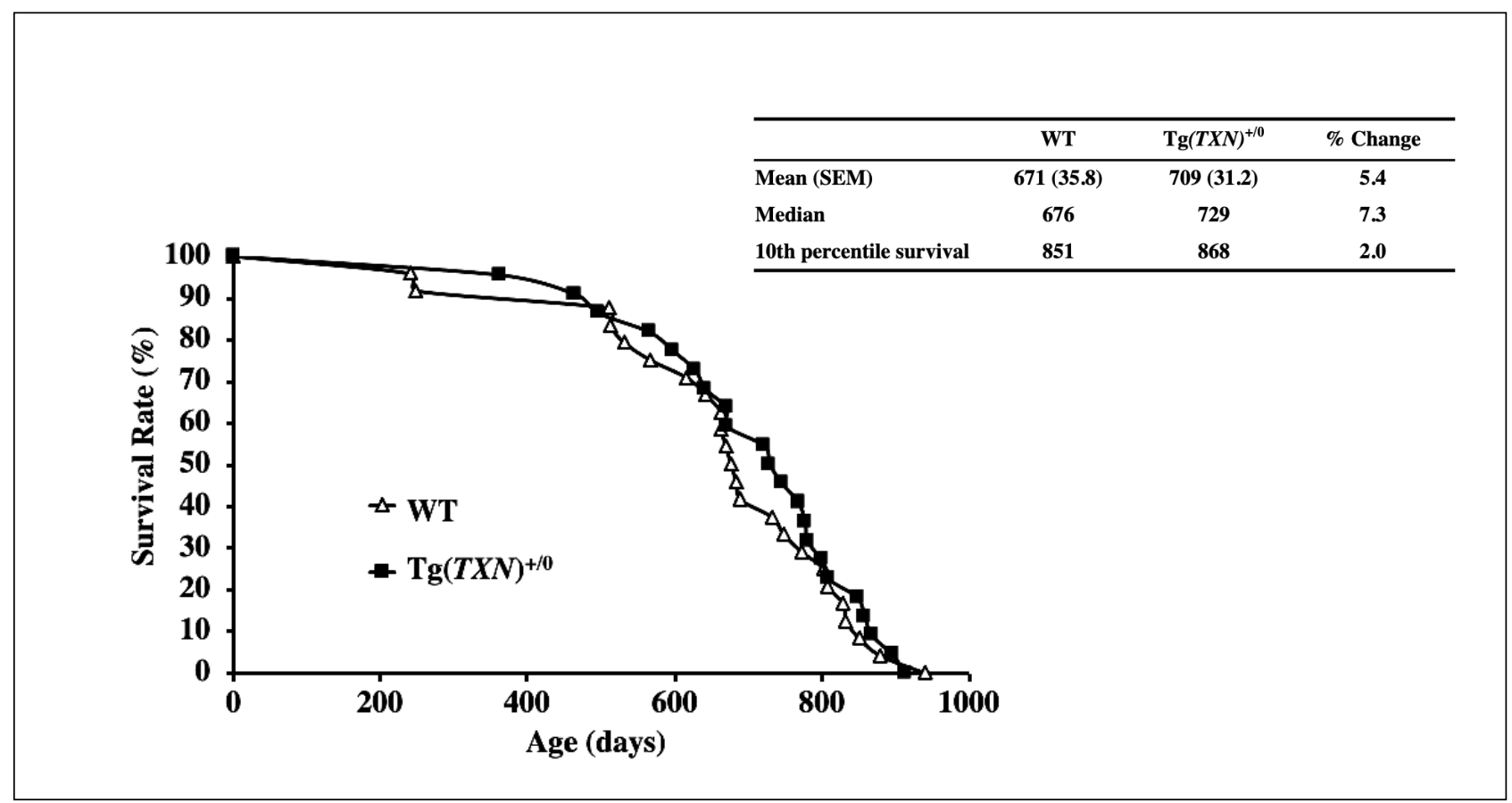

Figure 1. Survival curves of $\operatorname{Tg}(\mathrm{TXN})^{+/ 0}$ and WT rats. The results from a survival study with male $\operatorname{Tg}(\mathrm{TXN})^{+/ 0}$ (closed squares) and $\mathrm{WT}(\mathrm{open}$ triangles) rats are presented. The survival study was conducted with $22 \operatorname{Tg}(\mathrm{TXN})^{+/ 0}$ and $24 \mathrm{WT}$ male rats. No significant difference between $\operatorname{Tg}(\mathrm{TXN})^{+/ 0}$ and WT rats was observed.

cate that $\operatorname{Trx} 1$ overexpression is beneficial in young mice and has potential deleterious effects in old mice, possibly due to the development of different pathophysiological conditions. Similarly, overexpression of Trx1 in a line of transgenic rats had little effect on lifespan.

\section{Trx2 transgenic mice overexpressing thiore- doxin in mitochondria}

The results of the aging study with $\operatorname{Trx} 1$ transgenic mice suggest another possibility, in which Trx overexpression in mitochondria could be more important in aging. Indeed, there are several lines of evidence that strongly suggest that maintaining mitochondrial functions could provide benefits on age-related pathophysiological changes. For example, a study with mice overexpressing catalase in mitochondria (mCAT) showed significant extension of lifespan and attenuated development of some cancers, despite catalase overexpression in the nucleus or peroxisome ing Trx2 transgenic mice.

$\operatorname{Tg}(\mathrm{TXN} 2)^{+/ 0}$ mice were generated with the human thioredoxin 2 (TXN2) gene [23]. The young $\operatorname{Tg}(\mathrm{TXN} 2)^{+/ 0}$ mice showed significantly higher levels of Trx 2 in tissues examined than WT mice, which was maintained up to 22-24 months old [23]. $\operatorname{Tg}(\mathrm{TXN} 2)^{+/ 0}$ mice showed less hydrogen peroxide production from isolated mitochondria than WT control mice. Despite the reduced hydrogen peroxide production from mitochondria by Trx 2 overexpression, the effects on oxidative damage in the tissues were disappointing: 1) $\operatorname{Tg}(\mathrm{TXN} 2)^{+/ 0}$ mice showed slightly less (approximately 13-14\%) lipid peroxidation measured by plasma isoprostane levels than WT littermates, and 2) levels of 8-oxodG, a marker of DNA oxidation, were not changed by $\operatorname{Tr} x 2$ overexpression. The effects of Trx 2 overexpression on signaling pathways were also minimal: 1) mTOR and NFKB pathways were not changed in $\operatorname{Tg}(\mathrm{TXN} 2)^{+/ 0}$ mice compared to WT littermates; 2) Trx2 overexpression increased levels of c-Jun and c-Fos compared to WT mice [23]. 
Our survival study showed that male $\operatorname{Tg}(\mathrm{TXN} 2)^{+/ 0}$ mice had approximately $8-9 \%$ extension of lifespans (the mean, median, and 10th percentile) compared to WT control mice. However, these differences were not statistically significant [23]. The cross-sectional pathology results showed that male $\operatorname{Tg}(\mathrm{TXN} 2)^{+/ 0}$ mice had a similar number of total tumors (tumor burden) and incidence of lymphoma compared to WT mice. Although the incidence of lymphoma was not changed by $\operatorname{Trx} 2$ overexpression, $\operatorname{Tg}(\mathrm{TXN} 2)^{+/ 0}$ mice showed a slightly higher severity of lymphoma than WT mice [23]. These pathological analyses suggest that overexpression of Trx 2 in mitochondria may accelerate age-related lymphoma development, which is similar to the effects of Trx1 overexpression on tumor development $[15,16]$. As mentioned above, $\operatorname{Tg}(\mathrm{TXN} 2)^{+/ 0}$ mice showed higher levels of c-Jun and c-Fos than WT littermates. Activator protein 1 (AP-1), a complex of proteins of the Jun and Fos families, binds to TPA-response elements (TRE) or AP-1 binding sites to transcriptionally activate effector genes; subsequently, stimulation of cell proliferation and transformation occur. Thus, increased levels of c-Jun and c-Fos may be one of the mechanisms that accelerates agerelated tumor development in $\operatorname{Tg}(\mathrm{TXN} 2)^{+/ 0}$ mice.

\section{Effects of Trx down-regulation in the cytosol (Trx1) or mitochondria (Trx2) on aging}

We also conducted the survival studies to test the effects of thioredoxin down-regulation in the cytosol (Trx1) or mitochondria (Trx2) on aging. Because Trx 1 or Trx 2 null mice are embryonically lethal $[24,25]$, we conducted survival studies with heterozygous Trx1KO and heterozygous Trx2KO mice. Since Trx plays important roles in maintaining a reduced state in the cells, we anticipated that these mice might have higher oxidative stress/damage and/or mitochondrial functional changes, which may lead to a shorter lifespan.

Trx1 heterozygous knockout mice were generated with an ES clone purchased from Lexicon Pharmaceuticals, Inc. (OST452454), in which Trx1 expression was abolished by gene-trap insertion into the first intron of the Trx 1 gene. The young (4-6 months) and old (18-20 months) heterozygous Trx $1 \mathrm{KO}$ showed significantly lower (approximately $40 \%$ to $60 \%$ ) Trx 1 levels in the tissues examined compared to WT mice. Trx1 down-regulation did not affect levels and activities of other antioxidant enzymes. Trx1KO mice showed significantly lower levels of TrXASK1 complex and higher levels of ASK1 phosphorylation, which indicates that apoptosis is enhanced through the ASK pathway. As a consequence, a slight reduction in tumors was observed in Trx $1 \mathrm{KO}$ compared to WT mice at 22-24 months of age. However, Trx $1 \mathrm{KO}$ mice did not have remarkable changes in the survival curve compared to WT control mice, i.e., no significant changes were observed in median (50\% survival) and maximum (10\% survival) lifespans compared to WT mice (Figure 2).Therefore, a reduction in Trx 1 levels does not seem to have significant effects on aging, although there was a subtle indication it may attenuate age-related tumor development.

To test the effects of Trx down-regulation in the mitochon-

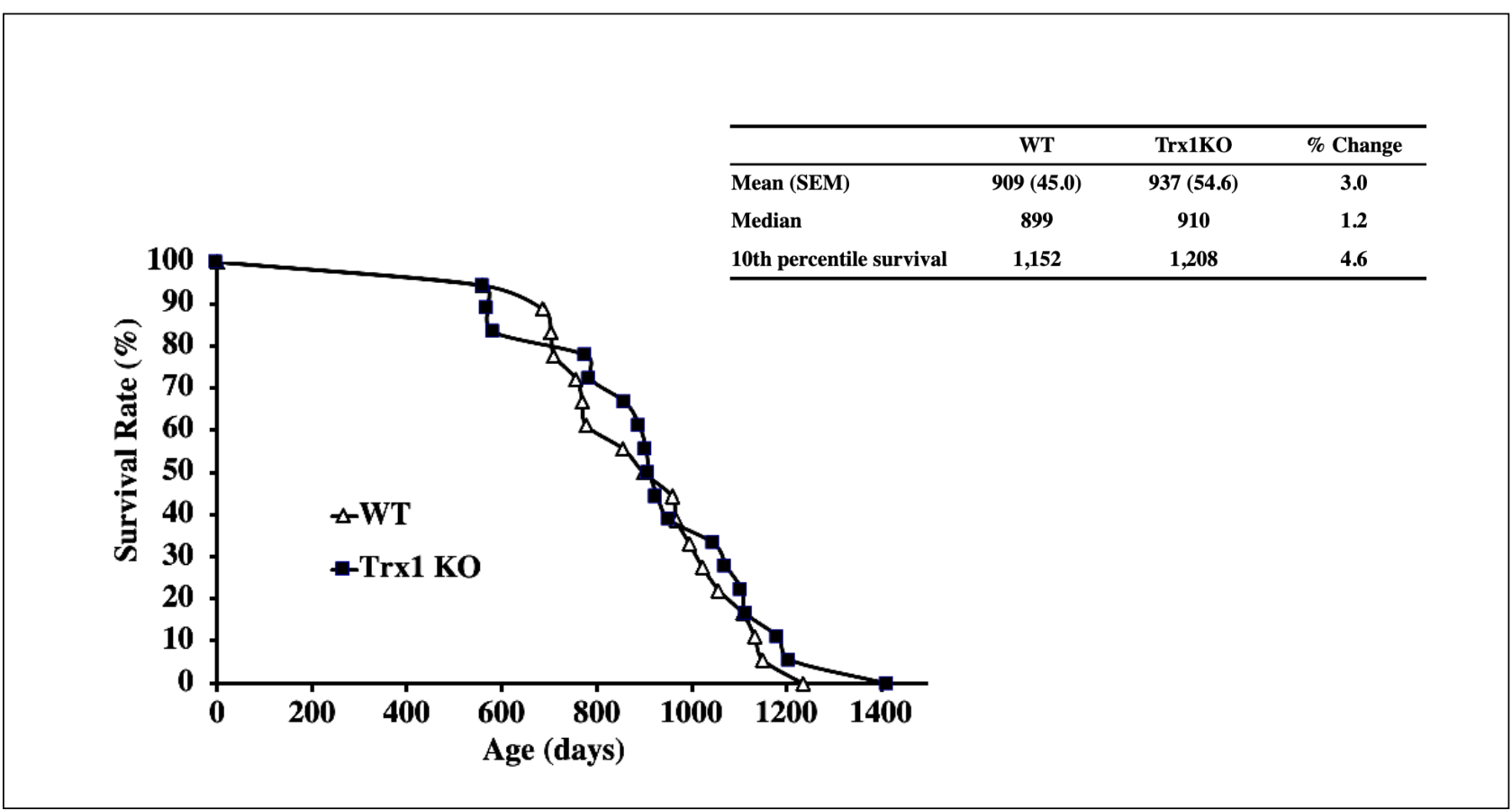

Figure 2. Survival curves of Trx1KO and WT mice. The survival curves of Trx1KO (closed squares) and WT (open triangles) mice are presented. The survival study was conducted with 18 Trx 1 KO mice and 18 WT male mice. No significant difference was observed between Trx 1 KO and WT mice. 
dria on aging, we conducted a survival study using Trx2KO mice [26]. Trx2KO mice were generated by gene trapping using random insertional mutagenesis [27]. Our previous study showed that levels of Trx 2 were approximately $50 \%$ less in Trx2KO mouse tissues compared to WT littermates [26]. Trx2 down-regulation did not change other antioxidant enzymes levels and activities. Reduced Trx in mitochondria showed that mitochondrial ATP production and ETC activity were significantly reduced and ROS production and oxidative damage were increased in Trx $2 \mathrm{KO}$ mice. In addition, the mitochondrial apoptosis pathway was enhanced in Trx2KO mice, resulting in higher sensitivity to apoptosis induction in isolated cells from Trx $2 \mathrm{KO}$ mice [26]. Although reduced Trx in mitochondria led to mitochondrial function impairment, increased ROS production and oxidative damage, and enhanced apoptosis in Trx2KO mice, the survival curves of Trx2 mice showed no significant changes compared to WT littermates [26, 28].

Thus, in spite of several pathophysiological changes, reduced Trx in either cytosol or mitochondria alone did not have a significant impact on the longevity of Trx $1 \mathrm{KO}$ or Trx2KO mice.

\section{Combined effects of overexpressing Trx1 and Trx2}

Based on our survival studies, overexpression of Trx 1 or Trx2 alone did not show a significant impact on longevity (i.e., extending the lifespan only in the earlier part of life by Trx 1 or slight extension of lifespan by Trx2), despite interesting cellular and physiological changes. These results indicate that Trx overexpression in only one compartment of the cell is not enough to extend the lifespan. In other words, Trx overexpression in both the cytosol and mitochondria may be required to have a notable impact on aging, i.e., significant life extension and reducing agerelated pathology including cancer.

Therefore, we examined the effects of overexpression of both Trx 1 and Trx 2 on lifespan and age-related pathology (especially cancer) with mice overexpressing Trx in both the cytosol and mitochondria (TXNTg x TXN2Tg) [29]. Our study showed that TXNTg x TXN2Tg mice overexpressed Trx 1 (2 to 14-fold increase) and Trx2 (1.8 to 2.3 -fold increase) in all tissues over the lifespan without changes in other antioxidant enzymes levels and activities or developmental abnormalities [29]. Contrary to our expectations, male TXNTg x TXN2Tg mice had significantly shorter lifespans compared to WT littermates, i.e. approximately $14.6 \%$ (mean), $16.3 \%$ (median), and $7 \%$ (10th percentile) shorter lifespans were observed in TXNTg $x$ TXN2Tg compared to WT mice [29]. The cross-sectional pathology demonstrated that TXNTg x TXN2Tg mice had slightly higher incidences of total neoplastic diseases and lymphoma than WT mice. TXNTg x TXN2Tg mice showed significantly higher grades of lymphoma compared to WT mice, which indicates that overexpression of Trx in both the cytosol and mitochondria accelerates lym- phoma development during aging [29].

The shorter lifespan and accelerated tumor development in TXNTg $x$ TXN2Tg mice were accompanied with changes in several redox-sensitive signaling pathways that could play important roles in: 1) cancer growth (HIF$1 \alpha$ levels and NFKB activity increase) [30-33] and 2) apoptosis suppression and cancer development (reduced ASK1 activity) [17]. These results were surprising since our studies with mice overexpressing Trx in the cytosol (Trx1) or mitochondria (Trx2) alone showed protection of cells/tissues against oxidative stress, and no deleterious effects were observed during aging in transgenic mice upregulating Trx1 or Trx2 $[15,16,23]$. Thus, to our knowledge, our study with TXNTg x TXN2Tg mice was the first report demonstrating the deleterious effects on aging by synergetic overexpression of Trx in both the cytosol and mitochondria.

\section{Combined effects of down-regulating Trx1 and Trx2}

Because of the shortened lifespan and enhanced tumor development observed in TXNTg x TXN2Tg mice, we subsequently tested whether down-regulation of Trx in both the cytosol and mitochondria affects aging and agerelated diseases in mice. Interestingly, survival studies using Trx1KO x Trx2KO mice showed opposite results from TXNTg x TXN2Tg mice: an approximately $10 \%$ and $9 \%$ extension of median lifespan in male and female Trx $1 \mathrm{KO}$ $\mathrm{x}$ Trx2KO mice compared to WT littermates, respectively. The detailed cross-sectional pathological analysis is ongoing, and we are currently examining several signaling pathways that play important roles in aging and cancer. Based on our preliminary data, the severity of lymphoma and disease burden (total number of histological changes/ body) seem to be lower in $\operatorname{Tr} 1 \mathrm{KO} \times \mathrm{Trx} 2 \mathrm{KO}$ mice compared to WT mice. Since other antioxidant enzymes levels/activities were similar between Trx $1 \mathrm{KO} \times \mathrm{Trx} 2 \mathrm{KO}$ and WT mice, these data suggest that synergetic downregulation of $\operatorname{Trx} 1$ and $\operatorname{Trx} 2$ had unexpected beneficial effects on aging. This observation is similar to the results reported with naked mole-rats and GPx4+/- mice [34, 35]. The slight extension (9-10\%) of lifespan and attenuated tumor development in Trx $1 \mathrm{KO} \times \mathrm{Trx} 2 \mathrm{KO}$ mice were accompanied with several signaling/molecular changes: 1) enhanced ASK1 phosphorylation; 2) increased both caspase activity and cytochrome c (cyt c) release; 3 ) reduced mTOR and HIF-1 $\alpha$; and 4) enhanced autophagy compared to WT mice. All of these pathways play important roles in cell death and growth, especially in cancer.

Based on our ongoing work and preliminary observations, we predict that reduced Trx levels in both the cytosol and mitochondria may reduce/retard age-related cancer development through: 1) enhanced removal of damaged cells (apoptosis) through mitochondrial and ASK1 pathways; and 2) enhanced removal of cellular damage (autophagy). Both of these processes could be major contributing fac- 
tors in reducing age-related genomic instability and attenuating age-related cancer development in Trx $1 \mathrm{KO} x$ Trx2KO mice. We also predict that reduced cancer development could further extend healthspan and lifespan in Trx1KO x Trx2KO mice. We are currently seeking the underlying mechanisms of the anti-cancer effects of reduced Trx in both the cytosol and mitochondria using a unique mouse model.

What have we learned from the survival studies with mice overexpressing or down-regulating Trx1 and/or Trx2, and where do we go from here?

Although the pathophysiological roles of Trx and thioredoxin interacting protein (Txnip) in mammals have been demonstrated $[1,36]$, the systemic examination of the effects of Trx on mammalian aging had not been achieved until our laboratory conducted the first systematic survival studies with mice overexpressing or down-regulating Trx1 and Trx2. Based on our data, several interesting features of thioredoxin and aging have been revealed: 1) overexpression of $\operatorname{Trx} 1$ alone $\left[\operatorname{Tg}(\text { act-TRX } 1)^{+/ 0}\right.$ and $\operatorname{Tg}(\mathrm{TXN})^{+/ 0}$ mice] extended lifespan only in the first half of life; 2) overexpression of $\operatorname{Trx} 2$ alone $\left[\operatorname{Tg}(\mathrm{TXN} 2)^{+/ 0}\right.$ mice] showed a slight extension of lifespan; 3) Trx 1 or Trx 2 downregulation in Trx $1 \mathrm{KO}$ or Trx $2 \mathrm{KO}$ mice had little effect on lifespan; 4) synergetic overexpression of Trx1 and Trx2 (TXNTg x TXN2Tg mice) resulted in a shorter lifespan with accelerated tumor development; and 5) an ongoing study using mice down-regulating Trx 1 and Trx 2 (Trx1KO $\mathrm{x}$ Trx2KO) slightly extended lifespan and suppressed tumor development in both males and females. These results clearly indicate that synergetic changes (overexpression or down-regulation) in $\operatorname{Trx} 1$ and $\operatorname{Trx} 2$ are required to have significant impacts on longevity, although overexpression or down-regulation of Trx 1 or Trx 2 alone showed various molecular and cellular changes.

Considering the life-extending effects of overexpressing Trx 1 or Trx 2 alone (only in the earlier part of lifespan by $\operatorname{Tg}(\mathrm{TXN})^{+/ 0}$ mice or slight extension of lifespan by $\operatorname{Tg}(\mathrm{TXN} 2)^{+/ 0}$ mice $)$, the shorter lifespan and accelerated tumor development observed in TXNTg x TXN2Tg mice were unexpected results. TXNTg x TXN2Tg mice showed several signaling/molecular changes, i.e., lower phosphorylated ASK1, and increased succinate, HIF-1 $\alpha$, and NFผB p65 levels compared to WT mice, which could play important roles in promoting age-related cancer development, possibly leading to a shorter lifespan [29].

These unexpected but very intriguing results led us to question whether synergetic down-regulation of Trx 1 and Trx2 affects aging and age-related pathology in mice. Trx $1 \mathrm{KO} \times$ Trx $2 \mathrm{KO}$ mice showed an approximately $10 \%$ and $9 \%$ extension of median lifespan in male and female mice, respectively, compared to WT littermates. The preliminary cross-sectional pathological analysis showed that the severity of lymphoma and disease burden were signifi- cantly lower in Trx1KO x Trx2KO mice compared to WT mice. These data indicate that down-regulation of Trx in both the cytosol and mitochondria has anti-cancer effects, which could be one of the major contributing factors to slight lifespan extension, although these mice could have a potential increase in oxidative stress. These unexpected and opposite results observed in both TXNTg x TXN2Tg and Trx1KO x Trx2KO mice led us to the following question: why did Trx down-regulation in both the cytosol and mitochondria reduce cancer and have a slight extension of lifespan? We are currently conducting experiments to seek the underlying mechanisms that reduce genomic instability and tumor development by enhanced apoptosis and autophagy as consequences of the changes in signaling pathways by reduced Trx 1 and Trx 2 .

The results of these studies, including the ongoing study with Trx1KO x Trx2KO mice: 1) provide a major advance in our understanding and new aspect of oxidative stress and redox regulation on cancer and aging; 2) lead us to identify the key pathways (i.e., apoptosis and autophagy) that prevent accumulation of damaged cells/cellular damage and genomic instability, leading to reduced cancer formation; and 3) identify potential pharmacological interventions (inhibition of Trx and/or downstream signaling pathways) for new prevention and/or therapy targets to attenuate age-related cancer development and extend healthspan.

\section{Declarations}

Acknowledgements: We acknowledge the Pathology Core in the San Antonio Nathan Shock Center (P30AG013319) for pathological analyses.

Financial support and sponsorship: This research was supported by the VA Merit Review grant from the Department of Veteran Affairs (Y.I.), NIH grant AG13319 (Y.I.), The American Federation for Aging Research (AFAR) grant (Y.I.), and a grant from the Glenn Foundation (Y.I.).

Conflict of interest: Yuji Ikeno is a member of the Editorial Board of Aging Pathobiology and Therapeutics. All authors declare no conflict of interest and were not involved in the journal's review or desicions related to this manuscript.

\section{References}

1. Arnér E S J, Holmgren A. Physiological functions of thioredoxin and thioredoxin reductase. European Journal of Biochemistry, 2000, 267(20): 6102-6109.

2. Brot N, Weissbach L, Werth J, et al. Enzymatic reduction of protein-bound methionine sulfoxide. Proceedings of the National Academy of Sciences, 1981, 78(4): 21552158.

3. Brot N, Weissbach H. Peptide methionine sulfoxide reductase: biochemistry and physiological role. Peptide Science, 2000, 55(4): 288-296. 
4. Chae H Z, Kang S W, Rhee S G. Isoforms of mammalian peroxiredoxin that reduce peroxides in presence of thioredoxin. Methods in enzymology. Academic Press, 1999, 300: 219-226.

5. Chae H Z, Kim H J, Kang S W, et al. Characterization of three isoforms of mammalian peroxiredoxin that reduce peroxides in the presence of thioredoxin. Diabetes research and clinical practice, 1999, 45(2-3): 101-112.

6. Kim K, Kim I H, Lee K Y, et al. The isolation and purification of a specific" protector" protein which inhibits enzyme inactivation by a thiol/Fe (III)/02 mixed-function oxidation system. Journal of Biological Chemistry, 1988, 263(10): 4704-4711.

7. Levine R L, Berlett B S, Moskovitz J, et al. Methionine residues may protect proteins from critical oxidative damage. Mechanisms of ageing and development, 1999, 107(3): 323-332.

8. Tagaya Y, Maeda Y, Mitsui A, et al. ATL-derived factor (ADF), an IL-2 receptor/Tac inducer homologous to thioredoxin; possible involvement of dithiol-reduction in the IL-2 receptor induction. The EMBO journal, 1989, 8(3): 757-764.

9. Spyrou G, Enmark E, Miranda-Vizuete A, et al. Cloning and expression of a novel mammalian thioredoxin. Journal of Biological Chemistry, 1997, 272(5): 2936-2941.

10. Miranda-Vizuete A, González J C F, Gahmon G, et al. Lifespan decrease in a Caenorhabditis elegans mutant lacking TRX-1, a thioredoxin expressed in ASJ sensory neurons. FEBS letters, 2006, 580(2): 484-490.

11. Svensson M J, Larsson J. Thioredoxin-2 affects lifespan and oxidative stress in Drosophila. Hereditas, 2007, 144(1): 25-32.

12. Takagi Y, Mitsui A, Nishiyama A, et al. Overexpression of thioredoxin in transgenic mice attenuates focal ischemic brain damage. Proceedings of the National Academy of Sciences, 1999, 96(7): 4131-4136.

13. Mitsui A, Hamuro J, Nakamura $\mathrm{H}$, et al. Overexpression of human thioredoxin in transgenic mice controls oxidative stress and life span. Antioxidants and Redox Signaling, 2002, 4(4): 693-696.

14. Nakamura H, Mitsui A, Yodoi J. Thioredoxin overexpression in transgenic mice. Methods in enzymology, 2002, 347: 436-440.

15. Pérez V I, Cortez L A, Lew C M, et al. Thioredoxin 1 overexpression extends mainly the earlier part of life span in mice. Journals of Gerontology Series A: Biomedical Sciences and Medical Sciences, 2011, 66(12): 1286-1299.

16. Flores L C, Roman M G, Cunningham G M, et al. Continuous overexpression of thioredoxin 1 enhances cancer development and does not extend maximum lifespan in male C57BL/6 mice. Pathobiology of Aging \& AgeRelated Diseases, 2018, 8(1): 1533754.

17. Saitoh M, Nishitoh H, Fujii M, et al. Mammalian thioredoxin is a direct inhibitor of apoptosis signal-regulating kinase (ASK) 1. The EMBO journal, 1998, 17(9): 25962606.

18. Hsieh C C, Papaconstantinou J. Thioredoxin-ASK1 complex levels regulate ROS-mediated p38 MAPK pathway activity in livers of aged and long-lived Snell dwarf mice. The FASEB Journal, 2006, 20(2): 259-268.

19. Powis G, Mustacich D, Coon A. The role of the redox protein thioredoxin in cell growth and cancer. Free Radical Biology and Medicine, 2000, 29(3-4): 312-322.

20. Schriner S E, Linford N J, Martin G M, et al. Extension of murine life span by overexpression of catalase targeted to mitochondria. science, 2005, 308(5730): 1909-1911.

21. Widder J D, Fraccarollo D, Galuppo P, et al. Attenuation of angiotensin II-induced vascular dysfunction and hypertension by overexpression of thioredoxin 2. Hypertension, 2009, 54(2): 338-344.

22. Zhang H, Luo Y, Zhang W, et al. Endothelial-specific expression of mitochondrial thioredoxin improves endothelial cell function and reduces atherosclerotic lesions. The American journal of pathology, 2007, 170(3): 11081120.

23. Roman M G, Flores L C, Cunningham G M, et al. Thioredoxin overexpression in mitochondria showed minimum effects on aging and age-related diseases in male C57BL/6 mice. Aging Pathobiology and Therapeutics, 2020, 2(1): 20-31.

24. Matsui M, Oshima M, Oshima H, et al. Early embryonic lethality caused by targeted disruption of the mouse thioredoxin gene. Developmental biology, 1996, 178(1): 179-185.

25. Nonn L, Williams R R, Erickson R P, et al. The absence of mitochondrial thioredoxin 2 causes massive apoptosis, exencephaly, and early embryonic lethality in homozygous mice. Molecular and cellular biology, 2003, 23(3): 916-922.

26. Pérez V I, Lew C M, Cortez L A, et al. Thioredoxin 2 haploinsufficiency in mice results in impaired mitochondrial function and increased oxidative stress. Free Radical Biology and Medicine, 2008, 44(5): 882-892.

27. Zambrowicz B P, Friedrich G A, Buxton E C, et al. Disruption and sequence identification of 2,000 genes in mouse embryonic stem cells. Nature, 1998, 392(6676): 608611.

28. Pérez V I, Bokov A, Van Remmen H, et al. Is the oxidative stress theory of aging dead?. Biochimica et Biophysica Acta (BBA)-General Subjects, 2009, 1790(10): 10051014.

29. Cunningham G M, Flores L C, Roman M G, et al. Thioredoxin overexpression in both the cytosol and mitochondria accelerates age-related disease and shortens lifespan in male C57BL/6 mice. Geroscience, 2018, 40(5-6): 453-468.

30. Hoesel B, Schmid J A. The complexity of NF- $\kappa B$ signaling in inflammation and cancer. Molecular cancer, 2013, 12(1): 1-15.

31. Huber M A, Azoitei N, Baumann B, et al. NF- $\kappa$ B is essential for epithelial-mesenchymal transition and metastasis in a model of breast cancer progression. The Journal of clinical investigation, 2004, 114(4): 569-581.

32. Semenza G L. Targeting HIF-1 for cancer therapy. Nature reviews cancer, 2003, 3(10): 721-732.

33. Xia Y, Shen S, Verma I M. NF- $\kappa B$, an active player in hu- 
man cancers. Cancer immunology research, 2014, 2(9): 823-830.

34. Andziak B, O'Connor T P, Qi W, et al. High oxidative damage levels in the longest-living rodent, the naked molerat. Aging cell, 2006, 5(6): 463-471.

35. Ran $\mathrm{Q}$ Liang $\mathrm{H}$, Ikeno $\mathrm{Y}$, et al. Reduction in glutathione peroxidase 4 increases life span through increased sen- sitivity to apoptosis. The Journals of Gerontology Series A: Biological Sciences and Medical Sciences, 2007, 62(9): 932-942.

36. Yoshihara E, Masaki S, Matsuo Y, et al. Thioredoxin/ Txnip: redoxisome, as a redox switch for the pathogenesis of diseases. Frontiers in immunology, 2014, 4: 514.

Cite this article as: Roman M G, Flores L C, Cunningham G M, et al. Thioredoxin and aging: What have we learned from the survival studies?[J]. Aging Pathobiology and Therapeutics, 2020, 2(3): 126-133. 